\title{
Indirect Adaptive Control for Discrete-Time Nonlinear Systems based on T-S Fuzzy Model
}

\author{
Khouloud Elloumi \\ Control \& Energy management \\ Laboratory \\ University of Safx, Sfax \\ Engineering School, BP 1173, \\ 3038 Sfax,Tunisia
}

\author{
Mohamed Jemel \\ Control \& Energy management \\ Laboratory \\ University of Safx, Sfax \\ Engineering School, BP 1173, \\ 3038 Sfax,Tunisia
}

\author{
Mohamed Chtourou \\ Control \& Energy management \\ Laboratory \\ University of Safx, Sfax \\ Engineering School, BP 1173, \\ 3038 Sfax,Tunisia
}

\begin{abstract}
The main goal of this paper is to present an indirect adaptive fuzzy control of discrete-time non affine nonlinear systems with parametric variations. The synthesis of the state feedback control law is based on the Takagi-Sugeno (T-S) fuzzy models developed by a local description of the considered system. In the first step, the model parameters locally estimated by the fuzzy model are adjusted using gradient method. In the second step, the local control gain based on pole placement is computed. After that, the global state feedback control law is applied to the nonlinear system. Based on the Lyapunov stability theory, the asymptotic stability of the proposed state feedback adaptive fuzzy control method is studied to ensure the global stability of the system. To illustrate the performance of the proposed controller, inverted pendulum and two links robot manipulator arm are presented.
\end{abstract}

\section{Keywords}

Indirect adaptive control, T-S fuzzy model, Discrete-time nonlinear systems, Stability analysis.

\section{INTRODUCTION}

The control of nonlinear systems has been the subject of many research works [1]. Fuzzy systems have been successfully applied to many control problems because they do not need an accurate mathematical model of the system under control [2]. In fact, fuzzy control algorithms are introduced based on the T-S fuzzy model [3-6]. The basic idea of this method is to represent the complex nonlinear system by linear local models. Then, for each one a state feedback control law is calculated. Thereafter, the global control law can be obtained either by combining all local control laws [4]. This design, called Parallel Distributed Compensation (PDC), Simplicity is the major advantage of this method.

In the literature of fuzzy adaptive control, many authors are interested in affine continuous systems. They integrate other techniques like the sliding mode control to develop an update parameters law such as [7]. Some others are based on the recursive least square method to propose update laws[8].Wang and Tanaka have discussed the design and the stability of discrete Single-Input Single-Output fuzzy dynamic control systems. The main goal of this work is to propose an indirect adaptive algorithm to elaborate a suitable fuzzy control law for discretes non affines nonlinear systems based on T-S fuzzy models.

The paper is organized as follows: In Section 2, the description of the T-S fuzzy model is formulated. Section 3 presents the proposed scheme of an indirect adaptive fuzzy control. In section 4, the stability conditions of the proposed method are discussed. Section 5, two numerical examples are presented to check the performance of the studied approach. Finally, some conclusions are presented in the last section.

\section{PROBLEM STATEMENT}

Consider the following discrete-time nonlinear dynamic system:

$\left\{\begin{array}{l}x(k+1)=f(x(k), u(k)) \\ y(k)=g(x(k))\end{array}\right.$

where $f$ and $g$ are two nonlinear functions. $x(k)=\left[x_{1}(k) x_{2}(k) \ldots x_{n}(k)\right]^{T} \in \mathfrak{R}^{n}, \mathrm{u}(k) \in \mathfrak{R}^{m}$

and $y(k) \in \mathfrak{R}^{q}$ are the measurable state vector, the input vector and the system output vector, respectively at the discrete time $k$.

\subsection{Takagi-Sugeno Fuzzy Model}

The nonlinear system (1) can be represented by a TakagiSugeno fuzzy dynamic model. It is described by a linear local model for each fuzzy rule [4].

The $i^{\text {th }}$ rule of the fuzzy model has the following form:

$$
\begin{aligned}
& R^{i} \text { : if } z_{1}(k) \text { is } M_{i 1} \text { and } \ldots \text { and } z_{p}(k) \text { is } M_{i p} \\
& \text { then }\left\{\begin{array}{l}
x(k+1)=A_{i}(k) x(k)+B_{i}(k) u(k) \\
y(k)=C_{i}(k) x(k)
\end{array}\right. \\
& i=1 \ldots r
\end{aligned}
$$

$$
\begin{gathered}
\text { Here, }{ }^{M_{i p}} \text { is the fuzzy set and } r \text { is the number of } \\
\text { fuzzy rules, } A_{i}(k) \in \mathfrak{R}^{n \times n}, B_{i}(k) \in \mathfrak{R}^{n \times m} \text { and } C_{i}(k) \in \mathfrak{R}^{q \times n}
\end{gathered}
$$
are the state matrices, the input matrix and the output matrix, respectively. $z_{1}(k), \ldots z_{p}(k)$ are the premise variables. The global fuzzy model of the non stationnary system has the following form:

$$
\begin{aligned}
& x(k+1)=\sum_{i=1}^{r} \alpha_{i}(z(k))\left(A_{i}(k) x(k)+B_{i}(k) u(k)\right) \\
& y(k)=\sum_{i=1}^{r} \alpha_{i}(z(k))\left(C_{i}(k) x(k)\right)
\end{aligned}
$$




$$
\alpha_{i}(z(k))=\frac{w_{i}(z(k))}{\sum_{i=1}^{r} w_{i}(z(k))}, \quad 0<\alpha_{i}(z(k))<1,
$$

and $\sum_{i=1}^{r} \alpha_{i}(z(k))=1, \quad i=1,2 \ldots r$

where $w_{i}(z(k))$ is the attributed weight for each rule $R^{i}$

$w_{i}(z(k))=\prod_{j=1}^{r} \mu_{i, j}\left(z_{j}(k)\right), w_{i}(z(k))>0, i=1,2 \ldots r$

$\mu_{i, j}\left(z_{j}(k)\right)$ is the appartenance degree of the membership function to the fuzzy set $M_{i j}$.

The nonlinear mathematical model is assumed known. Different local models are obtained by linearization around several operating points. The Jacobian matrices could be obtained using linearization method[1]:

$$
A_{i}=\left.\frac{\partial f}{\partial x}\right|_{\left(x_{0 i}, u_{0 i}\right)}, B_{i}=\left.\frac{\partial f}{\partial u}\right|_{\left(x_{0 i}, u_{0 i}\right)}, C_{i}=\left.\frac{\partial g}{\partial x}\right|_{\left(x_{0 i}, u_{0 i}\right)}
$$

\subsection{State Space Feedback Fuzzy Control}

In the PDC design, each control rule is designed from the same basic rules as the fuzzy model. The $i^{t h}$ fuzzy rule of the feedback state space controller is given as follows:

$$
\begin{aligned}
& R_{c}^{i}: \text { if } z_{1}(k) \text { is } M_{i 1} \text { and } \ldots \text { and } z_{p}(k) \text { is } M_{i p} \\
& \text { then } u_{i}(k)=-K_{i} x(k) \quad i=1,2 \ldots r
\end{aligned}
$$

$K_{i}$ is the state feedback gain of the ${ }^{i t h}$ local model which is computed to place all closed-loop eigenvalues of local models inside the unit circle at the appropriate values.

The global nonlinear fuzzy controller is presented by:

$$
\begin{aligned}
u(k) & =-\frac{\sum_{i=1}^{r} w_{i}(z(k)) K_{i} x(k)}{\sum_{i=1}^{r} w_{i}(z(k))} \\
& =-\sum_{i=1}^{r} \alpha_{i}(z(k)) K_{i} x(k)
\end{aligned}
$$

by substituting (8) into (3) the global control in closed loop can be written as:

$$
\begin{aligned}
x(k+1) & =\sum_{i=1}^{r} \alpha_{i}(k)\left(\left(A_{i}(k)-B_{i}(k) \sum_{j=1}^{r} \alpha_{j}(k) K_{j}\right) x(k)\right) \\
& =\sum_{i, j=1}^{r} \alpha_{i, j}(k) G_{i, j} x(k)
\end{aligned}
$$

where $G_{i, j}=A_{i}-B_{i} K_{j} \quad i, j=1, \ldots, r$

\section{INDIRECT ADAPTIVE FUZZY COPNTROL}

In this section, the adaptive control design for the dynamic fuzzy system will be presented.

\subsection{Fuzzy Controller Design}

The indirect adaptive fuzzy control scheme is illustrated by fig1. The gain in the control law given by equation (8)is calculated using matrices $A_{i}$ and $B_{i}$ which are unknown and variable over time. So, based on the assumption that Fuzzy models are universal approximators [9], ${ }^{A_{i}}$ and $B_{i}$ can be replaced by their estimates $\hat{A}_{i}$ and $\hat{B}_{i}$

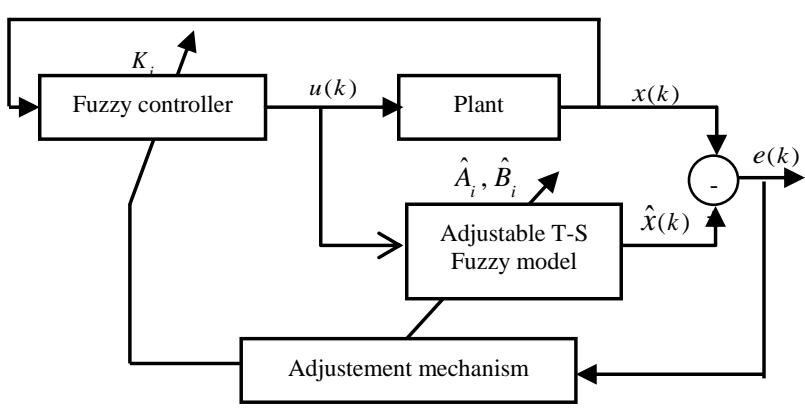

Fig 1: Indirect adaptive fuzzy control

\subsection{Estimation algorithm}

Adaptation laws are determined based on the gradient method to minimize the quadratic criterion $J(k)$. The error between the model and the plant is used to adjust on-line the parameters of the fuzzy model so that the error converges toward zero.

$J(k)=\frac{1}{2} e^{T}(k) e(k)$

The prediction error $e(k)$ is defined as:

$e(k)=\hat{x}(k+1)-x(k+1)$

$=\sum_{i=1}^{r} \alpha_{i}(k)\left(\hat{A}_{i}(k) x(k)+\hat{B}_{i}(k) u(k)\right)-x(k+1)$

Then, the following adaptation algorithm can be deduced:

$$
\begin{aligned}
& \hat{A}_{i}(k+1)=\hat{A}_{i}(k)-\varepsilon(k) \alpha_{i}(k) e(k) x^{T}(k) \\
& \hat{B}_{i}(k+1)=\hat{B}_{i}(k)-\varepsilon(k) \alpha_{i}(k) e(k) u^{T}(k) \\
& \varepsilon(k)=\frac{\delta(k)}{\sum_{i=1}^{r} \alpha_{i}^{2}(k)\left(x^{T}(k) x(k)+u^{T}(k) u(k)\right)}
\end{aligned}
$$

where $\tilde{A}_{i}$ and $\tilde{B}_{i}$ are the estimation errors matrices, defined as:

$\tilde{A}_{i}(k)=\hat{A}_{i}(k)-A_{i}(k)$

$\tilde{B}_{i}(k)=\hat{B}_{i}(k)-B_{i}(k)$

\subsection{Stability Analysis}

To demonstrate the stability of the closed-loop fuzzy control system which consists of the fuzzy model and the PDC controller, the following candidate Lyapunov function is defined: 
$V(k)=V_{1}(k)+V_{2}(k)+V_{3}(k)$

where

$V_{1}(k)=\|x(k)\|$

$V_{2}(k)=\sum_{i=1}^{r} \operatorname{tr}\left[\tilde{A}_{i}^{T}(k) \tilde{A}_{i}(k)\right]$

$V_{3}(k)=\sum_{i=1}^{r} \operatorname{tr}\left[\tilde{B}_{i}^{T}(k) \tilde{B}_{i}(k)\right]$

\section{- Theorem}

Consider the system described by (9), the control law (8) and the adaptation algorithm (13)-(15), a sufficient stability conditions for ensuring asymptotic stability of the closed loop follows:

$\sum_{i}^{r} \alpha_{i}(k) \lambda_{i \max }<1$

$0<\delta(k)<2$

\section{- Proof}

To ensure stability for the proposed scheme, the convergence conditions are obtained using the following inequalities:

$$
\Delta V_{1}(k)<0 \text { and } \Delta V_{2}(k)+\Delta V_{3}(k)<0
$$

For the first term, that concerns the dynamic fuzzy system expressed by (19), the demonstration of its stability condition is as follows:

$$
\Delta V_{1}(k)=V_{1}(k+1)-V_{1}(k)=\|x(k+1)\|-\|x(k)\|
$$$$
\Delta V_{1}(k)=\left\|\sum_{i, j}^{r} \alpha_{i, j}(k) G_{i, j} x(k)\right\|-\|x(k)\|
$$$$
\Delta V_{1}(k) \leq\left[\sum_{i, j}^{r} \alpha_{i, j}(k)\left\|G_{i, j}\right\|-1\right]\|x(k)\|
$$$$
\Delta V_{1}(k) \leq\left[\sum_{i}^{r} \alpha_{i}(k) \lambda_{i \max }-1\right]\|x(k)\|
$$

So $\Delta V_{1}(k)<0$ if $\sum_{i}^{r} \alpha_{i}(k) \lambda_{i \max }-1<0$

where ${ }^{\lambda}{ }_{\text {max }}$ is the maximum eigenvalues of the estimates of the matrix $G_{i, j}$ for $i, j=1 \ldots r$ given by expression (10)

By adopting the adaptation laws (13) and (14) and considering the equations (16) and (17), and by assuming that the system parameters are slightly variable through time, the following expressions are deduced:

$$
\begin{aligned}
& \tilde{A}_{i}(k+1)=\tilde{A}_{i}(k)-\alpha_{i}(k) \varepsilon(k) e(k) x^{T}(k) \\
& \tilde{B}_{i}(k+1)=\tilde{B}_{i}(k)-\alpha_{i}(k) \varepsilon(k) e(k) u^{T}(k)
\end{aligned}
$$

To manipulate (20) and (21), the following properties of trace are used: $\square \operatorname{tr}(A B)=\operatorname{tr}(B A)$ for any $A, B \in \mathfrak{R}^{n \times n}$

$\square \operatorname{tr}(A+B)=\operatorname{tr}(A)+\operatorname{tr}(B)$ for any $A, B \in \mathfrak{R}^{n \times n}$

$\square \operatorname{tr}\left(y x^{T}\right)=x^{T} y \quad$ for any $A, B \in \mathfrak{R}^{n \times 1}$

$$
\begin{aligned}
& \Delta V_{2}(k)=V_{2}(k+1)-V_{2}(k)= \\
& \sum_{i=1}^{r} \operatorname{tr}\left[\tilde{A}_{i}^{T}(k+1) \tilde{A}_{i}(k+1)\right]-\sum_{i=1}^{r} \operatorname{tr}\left[\tilde{A}_{i}^{T}(k) \tilde{A}_{i}(k)\right]
\end{aligned}
$$

$\Delta V_{2}=\sum_{i=1}^{r} \operatorname{tr}\left\{\left[\tilde{A}_{i}(k)-\alpha_{i}(k) \varepsilon(k) e(k) x^{T}(k)\right]^{T}\left[\tilde{A}_{i}(k)-\alpha_{i}(k) \varepsilon(k) e(k) x^{T}(k)\right]\right\}$

$-\sum_{i=1}^{r} \operatorname{tr}\left\{\tilde{A}_{i}^{T}(k) \tilde{A}_{i}(k)\right\}$

$\Delta V_{2}=-2 \sum_{i=1}^{r} \operatorname{tr}\left\{\tilde{A}_{i}^{T}(k) \alpha_{i}(k) \varepsilon(k) e(k) x^{T}(k)\right\}$

$+\sum_{i=1}^{r} t r\left\{\left[\alpha_{i}(k) \varepsilon(k) e(k) x^{T}(k)\right]^{T}\left[\alpha_{i}(k) \varepsilon(k) e(k) x^{T}(k)\right]\right\}$

$\Delta V_{3}(k)=V_{3}(k+1)-V_{3}(k)=\sum_{i=1}^{r} \operatorname{tr}\left[\tilde{B}_{i}^{T}(k+1) \tilde{B}_{i}(k+1)\right]$

$-\sum_{i=1}^{r} \operatorname{tr}\left[\tilde{B}_{i}^{T}(k) \tilde{B}_{i}(k)\right]$

$\Delta V_{3}=-2 \sum_{i=1}^{r}\left\{\alpha_{i}(k) \varepsilon(k) u^{T}(k) \tilde{B}_{i}^{T}(k) e(k)\right\}$

$+\sum_{i=1}^{r}\left\{\alpha_{i}^{2}(k) \varepsilon^{2}(k) u^{T}(k) e^{T}(k) e(k) u(k)\right\}$

$\Delta V_{2}+\Delta V_{3}=-2 \sum_{i=1}^{r}\left\{\alpha_{i}(k) \varepsilon(k)\left(x^{T}(k) \tilde{A}_{i}^{T}(k)+u^{T}(k) \tilde{B}_{i}^{T}(k)\right) e(k)\right\}$

$+\sum_{i=1}^{r}\left\{\alpha_{i}^{2}(k) \varepsilon^{2}(k)\left(x^{T}(k) e^{T}(k) e(k) x(k)+u^{T}(k) e^{T}(k) e(k) u(k)\right)\right\}$

Or $\sum_{i=1}^{r} \alpha_{i}(k)\left(x^{T}(k) \tilde{A}_{i}^{T}(k)+u^{T}(k) \tilde{B}_{i}^{T}(k)\right)=e^{T}(k)$

$\Delta V_{2}+\Delta V_{3}=-2 \sum_{i=1}^{r} \varepsilon(k) e^{T}(k) e(k)+$

$\sum_{i=1}^{r}\left\{\alpha_{i}^{2}(k) \varepsilon^{2}(k)\left(x^{T}(k) e^{T}(k) e(k) x(k)+u^{T}(k) e^{T}(k) e(k) u(k)\right)\right\}$

$\Delta V_{2}+\Delta V_{3}=\sum_{i=1}^{r}-e^{T}(k)[2 \varepsilon(k)-$ $\left.\alpha_{i}^{2}(k) \varepsilon^{2}(k)\left(x^{T}(k) x(k)+u^{T}(k) u(k)\right)\right] e(k)$

$\Delta V_{2}+\Delta V_{3}<0 \Leftrightarrow \Phi(k)=2 \varepsilon(k)$

$-\sum_{i=1}^{r} \alpha_{i}^{2}(k) \varepsilon^{2}(k)\left(x^{T}(k) x(k)+u^{T}(k) u(k)\right)>0$ 


$$
\begin{aligned}
& \Leftrightarrow\left\{\begin{array}{l}
\varepsilon(k)>0 \\
2-\sum_{i=1}^{r} \alpha_{i}^{2}(k) \varepsilon(k)\left(x^{T}(k) x(k)+u^{T}(k) u(k)\right)>0
\end{array}\right. \\
& \Leftrightarrow\left\{\begin{array}{l}
\varepsilon(k)>0 \\
\varepsilon(k)<\frac{2}{\sum_{i=1}^{r} \alpha_{i}^{2}(k)\left(x^{T}(k) x(k)+u^{T}(k) u(k)\right)}
\end{array}\right. \\
& \Leftrightarrow 0<\delta(k)<2
\end{aligned}
$$

\section{SIMULATION RESULTS}

To illustrate the performance of the presented approach, two nonlinear mechanical systems are considered as numerical example. The first one is the inverted pendulum which is widely used in the literature [1,4]. The second one is two degrees of freedom (DOF) robot manipulator arms, in order to validate our method in the case of a MIMO nonlinear system [1].

\subsection{Inverted pendulum}

\subsubsection{System Description}

The discrete dynamic model of the inverted pendulum on a cart is presented as following [10]:

$$
\left\{\begin{array}{l}
x_{1}(k+1)=x_{1}(k)+T_{e} x_{2}(k) \\
x_{2}(k+1)=x_{2}(k)+ \\
T_{e} \frac{g \sin \left(x_{1}(k)\right)-0.5 a m l\left(x_{2}(k)\right)^{2} \sin \left(2 x_{1}(k)\right)+a \cos \left(x_{1}(k)\right) u(k)}{4 l / 3-a m l \cos ^{2}\left(x_{1}(k)\right)}
\end{array}\right.
$$

where: $a=1 /(m+M)$ and $T_{e}$ is the sampling time.

$x_{1}(k)$ denotes the angle in radium of the pendulum from the vertical and $x_{2}(k)$ is the angular velocity in rad/s. $m$ and $M$, which are supposed contain unknown uncertainties, are the mass of the pendulum and the one of the cart, respectively. $2 l$ is the length of the pendulum, and $u$ is the force applied to the cart.

\subsubsection{Indirect Adaptive fuzzy control}

The T-S fuzzy model of the inverted pendulum is developed from dynamic equation of the nonlinear system, the model is described by the following plant rules:

$$
\text { if } \mathrm{x}_{1}(k) \text { is } M_{i 1} \text { then } x(k+1)=A_{i} x(k)+B_{i} u(k), i=1 \ldots 2
$$

The initial values of parameters of $A_{i}$ and $B_{i}$ are:

$$
\begin{array}{ll}
A_{1}=\left[\begin{array}{cc}
1 & 0.01 \\
0.1709 & 1
\end{array}\right], & B_{1}=\left[\begin{array}{c}
0 \\
-0.0018
\end{array}\right] \\
A_{2}=\left[\begin{array}{cc}
1 & 0.01 \\
0.7685 & 1
\end{array}\right], & B_{2}=\left[\begin{array}{c}
0 \\
-0.2910^{-3}
\end{array}\right]
\end{array}
$$

The control gain of each local model is calculated using the estimated matrices, to place the closed loop poles inside the unit circle. The poles of each rules are fixed as:

$$
p_{1}=[0.7 \pm 0.0208 i] \text { and } p_{2}=\left[\begin{array}{ll}
0.8 & 0.6
\end{array}\right]
$$

The rules of the PDC controller are:

$$
\text { if } \mathrm{x}_{1}(k) \text { is } M_{i 1} \text { then } \mathrm{u}(k)=-K_{i} x(k) \text { for } i=1 \ldots 2
$$

Fig. 2-4 presents the simulation results of the evolution of the control signal and the state variables of the inverted pendulum.

The masses are initialized at $m=m_{0}=2 \mathrm{~kg}$ and the one of the cart $M=M_{0}=8 \mathrm{~kg}$. At the instant $k=20$, the masses values are changed as follow: $m=4 \mathrm{~kg}$ and $M=11 \mathrm{~kg}$.

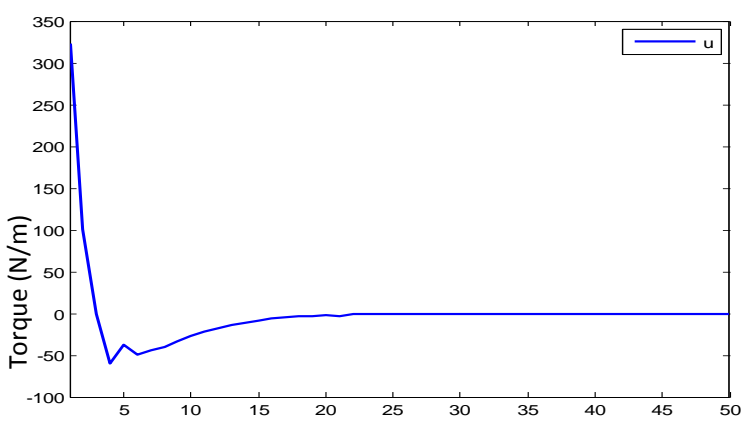

Fig 2: Evolution of the control signal
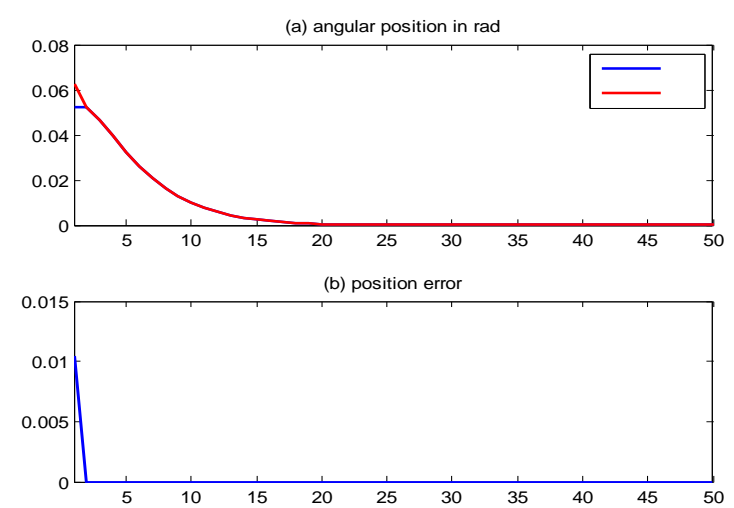

Fig 3: Evolution of ${ }^{x_{1}}$ and $\hat{x}_{1}$ (a) Evolution of the position error (b)
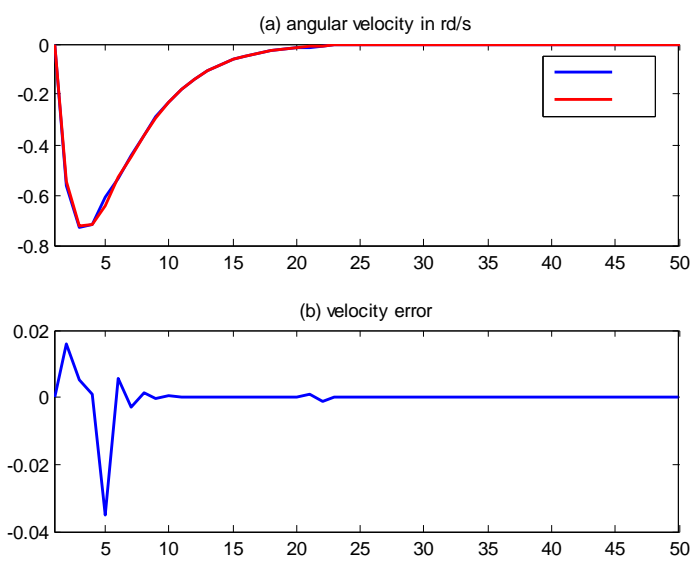

Fig 4: Evolution of $x_{2}$ and $\hat{x}_{2}$ (a)Evolution of the position error (b) 
Simulation results demonstrate that the proposed state space PDC controller guarantee the stabilization of the inverted pendulum to zero. The developed adaptive fuzzy control law is not sensible for the parameters variations.

\subsection{Two DOF robot arm}

\subsubsection{System Description}

The dynamic model of the system shown in Fig. 5 is given by the following equation [1]:

$$
M(q) \ddot{q}+C(q, \dot{q}) \dot{q}+G(q)=u
$$

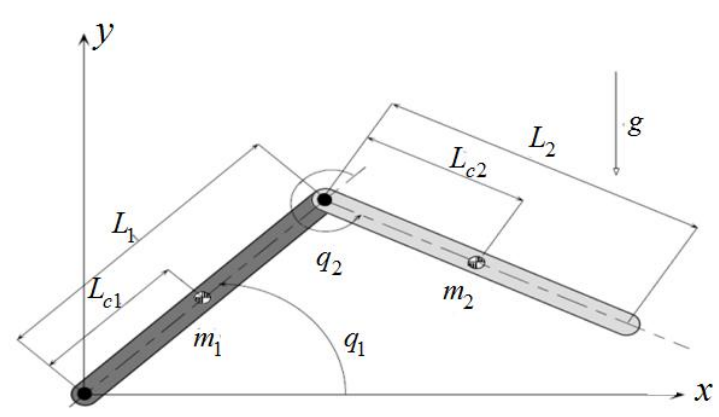

Fig 5: Two DOF robot arm

Where ${ }^{q}$ is an 2-dimensional vector of generalized coordinates representing joint positions, $u$ an 2-dimensional control (torque) input, and $M(q)$ a symmetric positive definite inertia matrix. The terms $C(q, \dot{q}) \dot{q}$ and $G(q)$ account for centrifugal/ Coriolis forces, and gravity.

where:

$M(q)=\left[\begin{array}{cc}a_{1}+2 a_{3} \cos \left(q_{2}\right) & a_{2}+a_{3} \cos \left(q_{2}\right) \\ a_{2}+a_{3} \cos \left(q_{2}\right) & a_{2}\end{array}\right]$

$C(q, \dot{q})=a_{3} \sin \left(q_{2}\right)\left[\begin{array}{cc}-\dot{q}_{2} & -\left(\dot{q}_{1}+\dot{q}_{2}\right) \\ \dot{q}_{1} & 0\end{array}\right]$

$G(q)=\left[\begin{array}{c}b_{1} \cos \left(q_{1}\right)+b_{2} \cos \left(q_{1}+q_{2}\right) \\ b_{2} \cos \left(q_{1}+q_{2}\right)\end{array}\right]$

and

$a_{1}=m_{1} L_{c 1}^{2}+m_{2} L_{c 2}^{2}+m_{2} L_{1}^{2}+I_{1}+I_{2}, a_{2}=m_{2} L_{c 2}^{2}+I_{2}$

$a_{3}=m_{2} L_{1} L_{c 2}, b_{1}=\left(m_{1} L_{c 1}+m_{2} L_{1}\right) g, \quad b_{2}=m_{2} L_{c 2} g$

$m_{h}, L_{h}$ and $I_{h}$ indicate the mass, the length and the moment of inertia of arm ${ }^{i}$ and $L_{c h}$ is the distance between the joint and the center of the arm respectively, for $h=1,2, g$ is the gravity constant. $m_{1}$ and $m_{2}$ are supposed to contain unknown uncertainties with known upper bound.

Let the sate vector and the input vector at the discrete time $k$ :

$$
\begin{aligned}
& x(k)=\left[x_{1}(k) x_{2}(k) x_{3}(k) x_{4}(k)\right]^{T}=\left[q_{1}(k) q_{2}(k) \dot{q}_{1}(k) \dot{q}_{2}(k)\right]^{T} \\
& u(k)=\left[u_{1}(k) u_{2}(k)\right]^{T}
\end{aligned}
$$

Suppose the matrix $H(x)=M^{-1}(x)=\left[\begin{array}{ll}h_{11} & h_{12} \\ h_{21} & h_{22}\end{array}\right]$

And the function

$$
F(x)=\left[\begin{array}{l}
f_{1}(x) \\
f_{2}(x)
\end{array}\right]=-M^{-1}(x)[C(x)+G(x)]
$$

Using the Euler approximation $x(k+1)=x(k)+T_{e} \dot{x}(k)$ with $T_{e}$ is the sampling time, the dynamic system can be described by following discrete equations:

$$
\left\{\begin{array}{l}
x_{1}(k+1)=x_{1}(k)+T_{e} x_{3}(k) \\
x_{2}(k+1)=x_{2}(k)+T_{e} x_{4}(k) \\
x_{3}(k+1)=x_{3}(k)+T_{e}\left[f_{1}(x)+h_{11}(x) u_{1}(k)+h_{12}(x) u_{2}(k)\right] \\
x_{4}(k+1)=x_{4}(k)+T_{e}\left[f_{2}(x)+h_{21}(x) u_{1}(k)+h_{22}(x) u_{2}(k)\right]
\end{array}\right.
$$

The parameters of the manipulator arms are: $m_{1}=m_{2}=1 \mathrm{~kg}$, $L_{1}=L_{2}=1 m, L_{c 1}=L_{c 2}=0.5 m, I_{1}=5, I_{2}=2$ and $g=9.8 \mathrm{~m} / \mathrm{s}^{2}$ and $T_{e}=0.01 \mathrm{~s}$.The angular positions $q_{1}, q_{2}$ are constrained within $\left[-\frac{\pi}{2}, \frac{\pi}{2}\right]$.

\subsubsection{Indirect Adaptive fuzzy control}

The basic idea is to stabilize this system around the origin using methods presented previously.

the manipulator states are assumed measurable and the mathematical model is considered known and variable. At $k=30$, the masses values are changed as follow: $m_{1}=1.5 \mathrm{~kg}$ and $m_{2}=1.5 \mathrm{~kg}$

two fuzzy rules are used for each state variable ${ }^{x_{1}}$ and ${ }^{x_{2}}$.

The fuzzy model rules are as following:

$$
\begin{aligned}
& \text { if } \mathrm{x}_{1}(k) \text { is } M_{i 1} \text { and } x_{2}(k) \text { is } M_{i 2} \\
& \text { then } x(k+1)=A_{i} x(k)+B_{i} u(k), i=1 \ldots 2
\end{aligned}
$$

The rules of the designed fuzzy controller are:

$$
\text { if } \mathrm{x}_{1}(k) \text { is } M_{i 1} \text { and } x_{2}(k) \text { is } M_{i 2}
$$$$
\text { then } \mathrm{u}(k)=-K_{i} x(k), i=1 \ldots 2
$$

The feedback gains $K_{i}$ are computed at each discrete time so that the closed loop poles are fixed inside the unit disc for all local models $\hat{A}_{i}-\hat{B}_{i} K_{j}$. The poles of each rule are:

$$
\begin{aligned}
& p_{1}=[0.1 \pm 0.1 i, 0.4 \pm 0.2 i] \\
& \text { and } p_{2}=[0.0016 \pm 0.3834 i,-0.0022 \pm 0.5879 i]
\end{aligned}
$$

Fuzzy rules consequence parameters are initialized to satisfy the system controllability condition. The adjustment parameter in the adaptation laws (13)-(15) is variable between 0 and 2. 
Figs. 6-10 present the simulation results of behavior of the state variables of the robot manipulator arms with parametric variation and its control laws respectively.

Simulation results demonstrate that the proposed state space controller is able to stabilize the robot manipulator from initial conditions in the origin in spite of presence of mass variations.
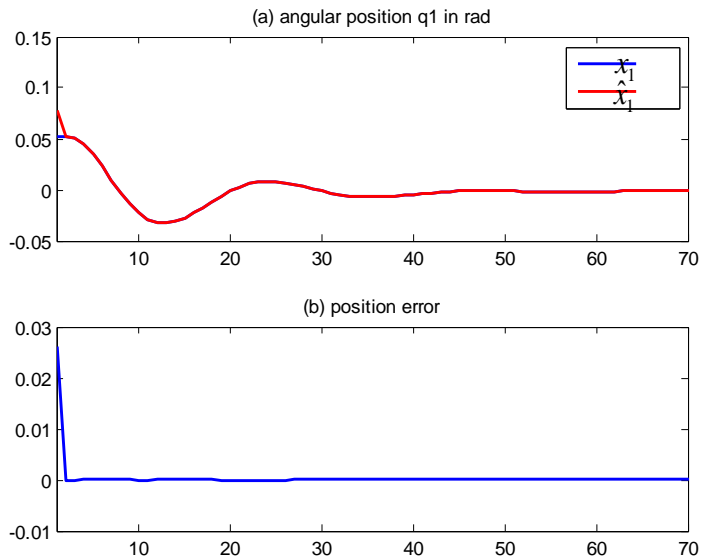

Fig 6 : Evolution of $x_{1}$ and $\hat{x}_{1}$ in rad (a)Evolution of position error of the first link (b)
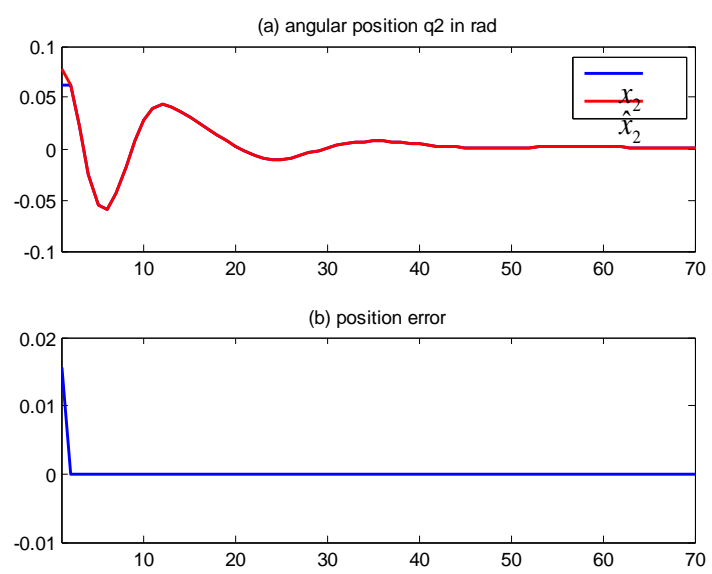

Fig 7: Evolution of $x_{2}$ and $\hat{x}_{2}$ in rad (a) Evolution of position error of the second link (b)

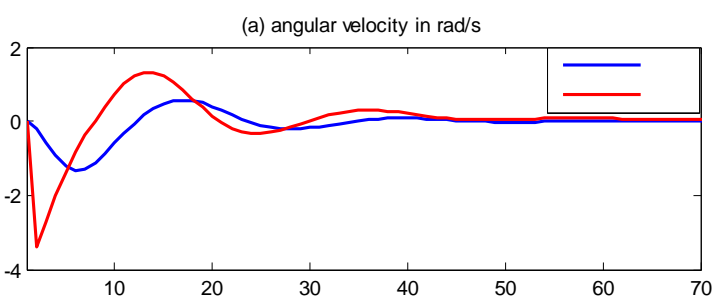

(b) velocity error

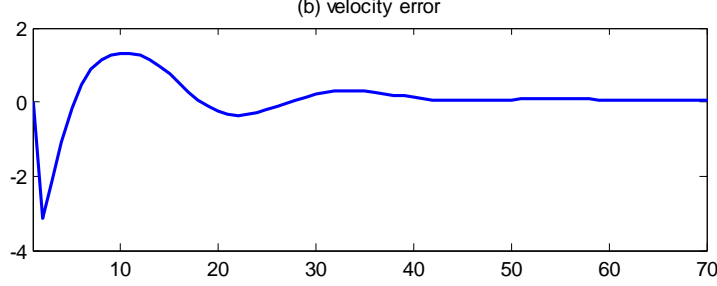

Fig 8: Evolution of $x_{3}$ and $\hat{x}_{3}$ in $\mathrm{rad} / \mathrm{s}$ (a)Evolution of velocity error of the first link (b) $\hat{x}_{4}$
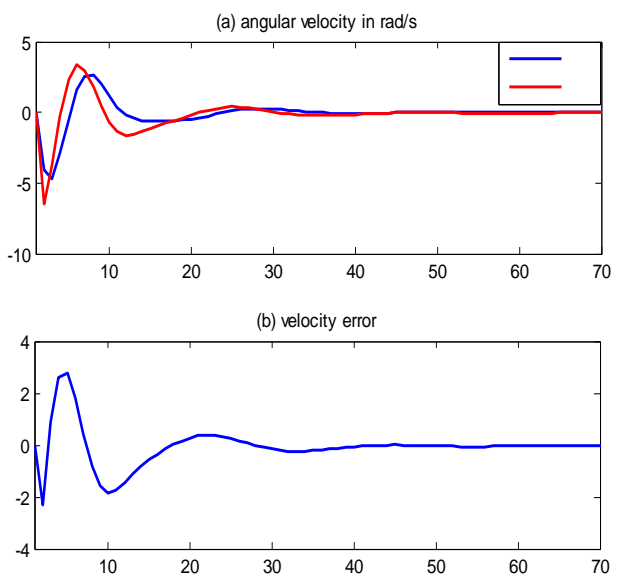

Fig 9: Evolution of $x_{4}$ and $\hat{x}_{4}$ in rad/s (a)Evolution of velocity error of the second link (b)

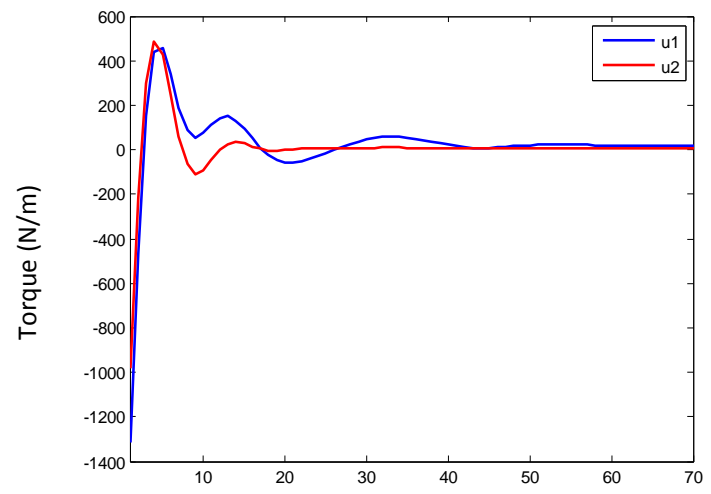

Fig 10: Evolution of the control signal

\section{CONCLUSIONS}

This work deals with indirect adaptive fuzzy control by state feedback control with PDC technique for non affine nonlinear systems. The control scheme is applied for the stabilization of variable system. At the first step, the parameters of the fuzzy model under an adaptation law are estimated. In the second step, the control law is computed basing on pole placement method. Satisfactory results simulations, demonstrate the ability of the control law, based on the adaptation algorithm, to stabilize the system even in the presence of parameter variations. Robust study can be the object of future works.

\section{REFERENCES}

[1] Khalil, H.K. 2015. Nonlinear Control, $1^{\text {st }}$ edition 07458. Pearson education, Prentice Hall.

[2] Boulkroune, A. and al. 2014. Indirect adaptive fuzzy control scheme based on observer for nonlinear systems:A novel SPR-filter approach, Neurocomputin.

[3] Slotine, J. J. and Li, W. 1991. Applied Nonlinear Control, Prentice Hall Englewood Cliffs, New Jersey.

[4] Tanaka, K. and Wang, H. O. 2001. Fuzzy Control Systems Design and Analysis, John Wiley \& Sons.

[5] Benhlima, D. 2009. "Méthodologies de commande Floue adaptative de certaines classes de systèmes non 
linéaires". Doctoral thesis, Sfax Engineering School, Sfax, Tunisia.

[6] Moraire, Y. 2001. "Mise en oeuvre de lois de commande pour les modèles flous de type Takagi-Sugeno". Doctoral thesis, University of Valenciennes and du Hainaut Cambrésis.

[7] Su, J. P. and al. 2001. Adaptive fuzzy sliding mode control with GA-based reaching laws. Fuzzy Sets and Systems, vol. 120, pp. 145-158.
[8] Ratmüller, M. and Murgaš, J. 2004. Fuzzy modelling and adaptive control of uncertain system. Journal of Electrical Engineering, vol. 55, no. 9-10, pp. 251-255.

[9] Wang, L.X. 2002. Fuzzy basis function, universal approximation and orthogonal least squares learning. IEEE Transaction on Neural Networks. Vol. 3. Pp. 807814.

[10] Wang, L.X. 1996. Stable adaptive fuzzy controller with application to inverted pendulum tracking. IEEE transaction on systems, Man and Cybermetics. Vol. 26. Pp 677-69. 